\title{
MIRACLE's Hybrid Approach to Bilingual and Monolingual Information Retrieval
}

\author{
José M. Goñi-Menoyo ${ }^{1}$, José C. González ${ }^{1,3}$, J.L. Martínez-Fernández ${ }^{2,3}$, \\ and J. Villena ${ }^{2,3}$ \\ ${ }^{1}$ Universidad Politécnica de Madrid \\ ${ }^{2}$ Universidad Carlos III de Madrid \\ ${ }^{3}$ DAEDALUS - Data, Decisions and Language, S.A. \\ josemiguel.goni@upm.es, jgonzalez@dit.upm.es, \\ joseluis.martinez@uc3m.es, julio.villena@uc3m.es
}

\begin{abstract}
The main goal of the bilingual and monolingual participation of the MIRACLE team in CLEF 2004 was to test the effect of combination approaches on information retrieval. The starting point was a set of basic components: stemming, transformation, filtering, generation of n-grams, weighting and relevance feedback. Some of these basic components were used in different combinations and order of application for document indexing and for query processing. A second order combination was also tested, mainly by averaging or selective combination of the documents retrieved by different approaches for a particular query.
\end{abstract}

\section{Introduction}

The MIRACLE team is made up of three university research groups located in Madrid (UPM, UC3M and UAM) along with DAEDALUS, a company founded in 1998 as a spin-off of two of these groups. DAEDALUS is a leading company in linguistic technologies in Spain ${ }^{1}$, and is the coordinator of the MIRACLE team. This is the second participation in CLEF, following that of 2003 [3], [6]. In addition to the bi- and monolingual tasks, the team participated in the ImageCLEF and Q\&A tracks.

The main purpose of the bi- and monolingual participation was to test the effect of combination approaches on information retrieval. The starting point was a set of basic components: stemming, transformation (transliteration, elimination of diacritics and conversion to lowercase), filtering (elimination of stop and frequent words), generation of n-grams, weighting (giving more importance to titles) and relevance feedback.

${ }^{1}$ DAEDALUS clients include leading companies in different sectors: media (EL PAÍS), publishing (Grupo SM), telecommunication (Grupo Telefónica), digital rights management (SGAE), photography (StockPhotos) and the reference institution for the Spanish language, Instituto Cervantes. Its portfolio of solutions includes STILUS ${ }^{\circledR}$ (professional spell, grammar and style checking of texts in Spanish), K-Site ${ }^{\circledR}$ (information retrieval, fuzzy search and knowledge management), LUCAS (universal locator of audiovisual contents, an Internet spider), etc. 
Some of these basic components are used in different combinations and order of application for document indexing and for query processing. A second order combination was also tested, mainly by averaging or by selective combination of the documents retrieved by different approaches for a particular query. When evidence is found of better precision of one system at one extreme of the recall level (i.e. 1,0), complemented by the better precision of another system at the other recall end (i.e. $0,0)$, then both are combined to benefit from their complementary results.

In addition, during the last year our group has been developing an indexing system based on the trie data structure [2]. Tries [1] are successfully used by the MIRACLE team for an efficient storage and retrieval of huge lexical resources, combined with a continuation-based approach to morphological treatment. However, the adaptation of these structures to manage efficiently document indexing and retrieval for commercial applications has been a hard task. The currently available prototype shows a great improvement in performance. (Both indexing and retrieval times are considerably reduced.) However, this system was not fully operational in this CLEF campaign. So, the Xapian [10] indexing system, robust, efficient, and well suited for our purposes, was used as in the last campaign.

For the 2004 campaign, runs were submitted for the following tracks:

a) Monolingual Russian.

b) Monolingual French.

c) Bilingual Dutch to French.

d) Bilingual German to French.

\section{Description of the Tools in MIRACLE's Tool Box}

The Xapian system was the basic indexing and retrieval tool for the bilingual and monolingual experiments by the MIRACLE group. Before indexing, document collections were pre-processed using different combinations of scripts, each one oriented to a particular experiment. For each of these, topic queries were also processed using the same combination of scripts. (Although some variants have been used, as will be described later).

The baseline approach to document and topic query processing is made up of the following sequence of steps:

1. Extraction: Ad-hoc scripts are run on the files that contain particular documents or topic query collections, to extract the textual data enclosed in XML tags. All those permitted for automatic runs were used. (Depending on the collection, all of the existing TEXT, TITLE, LEAD1, TX, LD, TI, or ST for document collections, and the contents of the TITLE and DESC fields for topic queries; NARR field contents were systematically ignored). The contents of these fields were concatenated to feed the following processing steps. However, in some experiments only the titles were extracted (including, in the run identifier, the strings titnormal, titnostem or titngrams), and in some normal experiments (see below) in monolingual Russian, the terms appearing within the TITLE fields were given more importance by repeating them several times (these experiments include the strings nomaltit1, nor- 
maltit 2 or normaltit 3 in the identifiers, when the titles terms are included one, two or three more times).

2. Parsing: A simple parsing process is followed to eliminate punctuation marks and detect basic indexing chunks (usually words, but some basic entities can also be detected, such as compounds, proper nouns, and so on). It is clear that the quality of this step is of paramount importance for precise document processing. A highquality entity recognition (proper nouns or acronyms for people, companies, countries, locations, and so on) could improve the precision and recall figures of the overall retrieval, as well as a correct recognition and normalization of dates, times, numbers, etc.

3. Lowercase words: All document words are normalized by converting all uppercase letters to lowercase.

4. Stopwords filter: All words recognised as stopwords are eliminated from the document. Stopwords in the target languages were initially obtained from [9], but were extended using several other sources and our own knowledge and resources.

5. Stemming: This is applied to each of the words in the document. The stemmer used is the one referenced in [7].

6. Remove accents: All document words are normalized by eliminating accents in stemmed words. Note that this process can be done before stemming, but the resulting lexemes are different. Despite this, in some experiments, this step was performed before stemming.

\section{Final use:}

a. Indexing: When all the documents processed through the former steps are ready for indexing, they are fed into a Xapian ad-hoc front-end to build the Xapian document database.

b. Retrieval: When all the documents processed by the former steps are topic queries, they are fed to a Xapian ad-hoc front-end to search the previously built Xapian index. In the 2004 experiments, only OR combinations of the search terms were used.

In the case of the Russian language, the basic processing steps described above are slightly changed, due to the different encodings of the Russian files and the resources used for Russian: while document collection and topics files were encoded in UTF8, as well as stopword resources, the stemming resources worked in KOI8, so some recoding steps were added at appropriate processing points. In addition to this, some other tools did not work properly with the UTF8 encoding, so others had to be added: (a) The parsing process was simplified even more, using a sed script to achieve basic punctuation processing, and (b) a transliteration of the files to the ASCII charset was needed to get the XAPIAN indexing system to work. The transliteration script used was the one available in reference [9].

In addition to the baseline (or normal) experiments (identified with the suffix normal in the run identifiers), other experiments were also defined as variations: If the stemming step was not carried out, the resulting experiments were labeled with the suffix nostem, where the actual word forms appearing in the documents are used for indexing and retrieval. A variant of the nostem experiments was also tested, where a set of $n$-grams was generated from each of the actual word forms in the documents. These experiments were labeled with identifiers of the form $n$ grams $X Y$, where $\mathrm{X}$ is the length of the n-grams and $\mathrm{Y}$ the number of characters that overlap between two 
consecutive n-grams. (For example, in an experiment referred to by an identifier with the suffix ngrams54, from president we would obtain the n-grams: "_pres", "presi", "resid", "eside", "siden", "ident", and "dent_". The symbol "_, is introduced to denote word boundaries. Note that four characters overlap between two consecutive ngrams).

In the case of the topic queries, an additional variation is introduced: the FW (Frequent Words) filter is applied by filtering out the 20 most frequent words, or stems, that appear in the corpora from the queries, as well as some typical query terms. These variants were identified by using the $F W$ string in the run identifier.

The Xapian engine allows us to use relevance feedback, so we use this technique in several experiments. When the terms of the first documents retrieved in the first retrieval step are fed back to a second retrieval step, we used the strings $R 1, R 2, R 3, R 4$ or $R 5$, in the run identifier depending on the actual number of documents used. Note that using relevance feedback does not affect the indexing processes, and can be applied in any of the variants used for processing the document collections or the topic queries.

For translation purposes, the SYSTRAN [10] system was used. Our tests carried out on the collections and topics of CLEF 2003, showed that SYSTRAN outperformed other on-line translators in the selected pairs of languages (Dutch to French and German to French) when used to find documents in the French collections from queries in Dutch or German. As other pairs such as Finnish and Swedish to French were not available on-line in SYSTRAN, other translators were tested with very poor results. So, no runs were prepared for them.

\section{Description of the Baseline Experiments}

Not all the possible combinations of the variants described in the previous section were tried in the experiments due to evident limitations of computing resources and time. The experiments were tried in a rather intuitive, non-systematic way, trying to test a wider and richer set of trials.

To compare these approaches, we used these techniques following the instructions given for CLEF 2003 (corpora and topic queries) and using the appropriate qrels available at the beginning of this campaign. The experiments that provided the best precision results in the CLEF 2003 scenario were selected for submission to CLEF 2004.

The appendix shows the baseline experiments, and the precision values obtained. It also shows those that were selected for submission to CLEF 2004, as well as the results obtained using the CLEF 2003 data.

In Figure 1, the results obtained using the best baseline experiments submitted to CLEF 2004 are compared with the results obtained by exactly the same system when applied to the 2003 tasks. The comparison shows qualitative differences between the 2003 and 2004 topics. No figure is presented for French, as all the submitted runs were, in this case, obtained through combination (see the next section). 

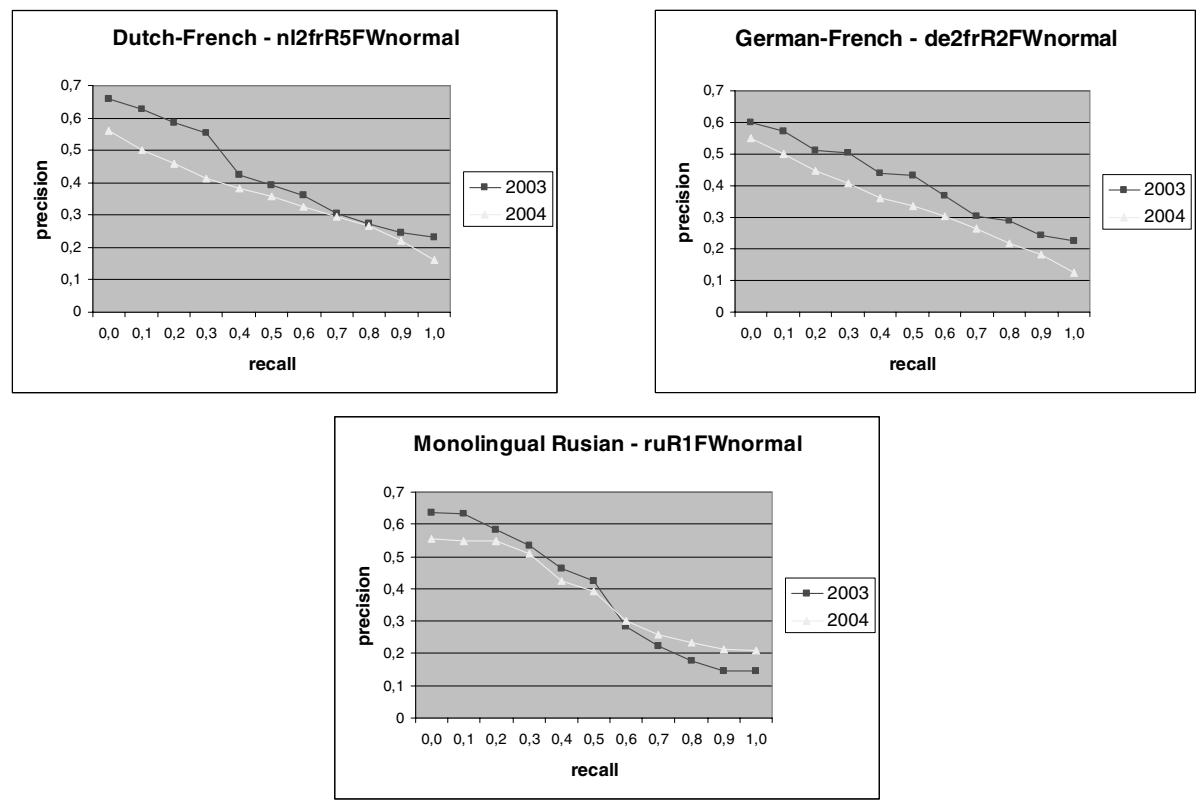

Fig. 1. Precision-recall curves for selected baseline experiments in CLEF 2003 and CLEF 2004

\section{Description of Combined Experiments}

In this campaign, some tests were carried out trying to combine the results from the basic experiments in different ways. The underlying hypothesis is that, to some extent, the documents with a good score in almost all experiments are more likely to be relevant than other documents that have a good score in one experiment but a bad one in others. Two strategies were followed for combining experiments:

- Average: The relevance figures obtained using the Xapian probabilistic retrieval in all the experiments to be combined for a particular document in a given query are added. This approach combines the relevance figures of the experiments without highlighting a particular experiment.

- Asymmetric DWX combination: In this particular type of combination, two experiments are combined in the following way: The relevance of the first $\mathrm{D}$ documents for each query of the first experiment is preserved for the resulting combined relevance, whereas the relevance for the remaining documents in both experiments are combined using weights $\mathrm{W}$ and $\mathrm{X}$. We have only run experiments labeled " 101 " and " 201 ", that is, the ones that get the one (or two) more relevant documents from the first basic experiment and all the remaining documents retrieved from the second basic experiment, re-sorting all the results using the original relevance.

Average combinations get better figures in average precision or in precisions at 0 or 1 points of recall than the original basic experiments. The reason could be that 
relevant documents that appear with a high score in the combined experiments are strengthened. Average combinations seem to improve results slightly, whereas asymmetric combinations do not. The particular experiments that were combined and the type of combination are shown in the appendix, for each one of the four tracks.

In Figure 2, the results obtained from the best combined experiments submitted to CLEF 2004 are compared with the results obtained using exactly the same systems when applied to the 2003 tasks. The comparison shows again the qualitative differences between the 2003 and 2004 topics.
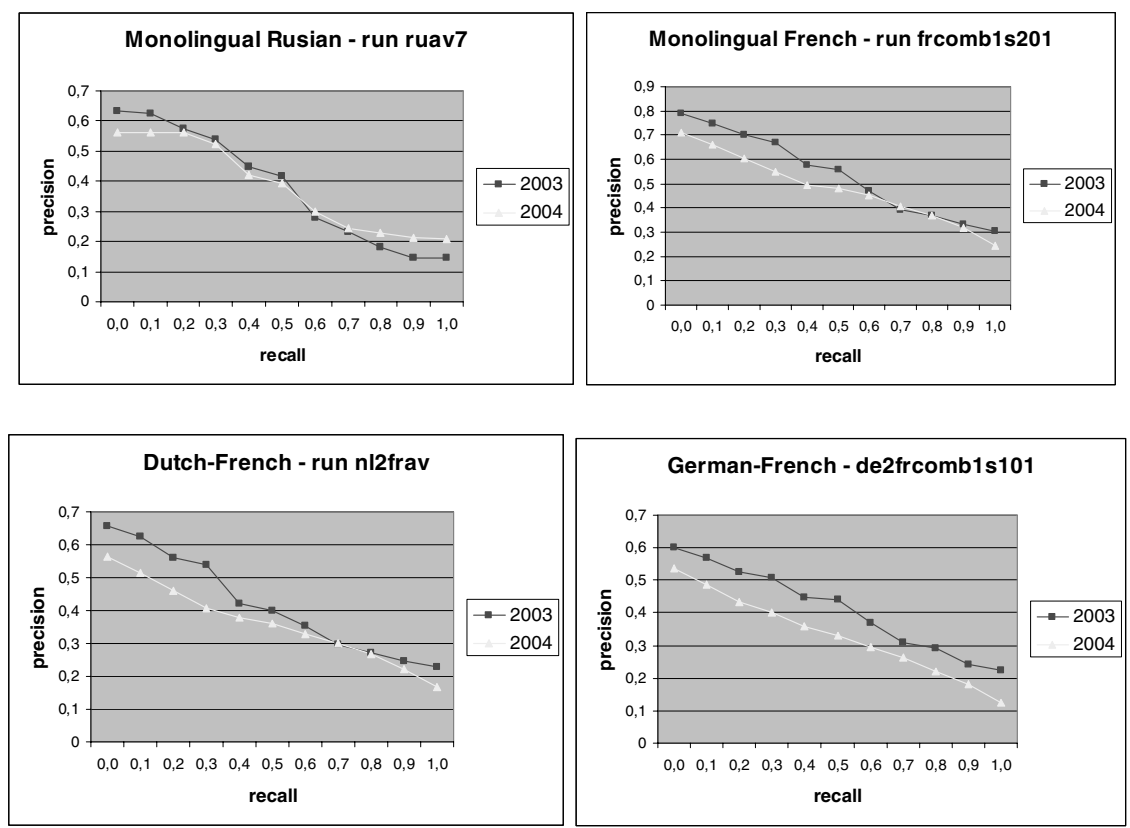

Fig. 2. Precision-recall curves for selected combined experiments in CLEF 2003 and CLEF 2004

\section{Conclusions}

The combination approach seems to improve the precision results slightly for IR retrieval tasks, although an in-depth analysis of the reasons for this is still needed. The differences shown between the 2003 and 2004 experiments seem to be highly idiosyncratic, depending to a great extent on the different topics selected each year. This is particularly true in the case of Russian, due to the low number of documents relevant for the topic set. Regarding the basic experiments, the general conclusions were known in advance: retrieval performance can be improved by using stemming, filtering of frequent words, appropriate weighting and relevance feedback with a few documents. On the other hand, n-grams performed worse than expected. 
Future work of the MIRACLE team in these tasks will be directed to several lines of research: (a) Getting better performance in the indexing and retrieval phases in order to be able to carry out experiments in a more efficient way (indexing times for the huge document collection is now excessive for a flexible scheduling of experiments). This will be achieved using our own trie-based libraries for the indexing and retrieval phases. (b) Improving the first parsing step; in our opinion, this is one of the most critical processing steps and can improve the overall results of the IR process. A good entity recognition and normalization is still missing in our processing scheme for these tasks.

\section{Acknowledgements}

This work has been partially supported by the OmniPaper project (European Union, 5th Framework Programme for Research and Technological Development, IST-200132174).

The participation of the MIRACLE team in CLEF 2004 was also partially funded by the Regional Government of Madrid through the research project "MIRACLE: Multilingual Information Retrieval System and its Evaluation under the CLEF European Initiative" (07T/0055/2003) and through its Entrepreneurship Innovation Programme (Madrid Innova, project PIE/594/2003).

Special mention must be done to our colleagues at other members of the MIRACLE group: Ana M. García-Serrano, Paloma Martínez-Fernández, César de Pablo-Sánchez, and Javier Alonso-Sánchez.

\section{References}

1. Aoe, Jun-Ichi, Morimoto, Katsushi, Sato, Takashi: An Efficient Implementation of Trie Structures. Software Practice and Experience 22(9) (1992) 695-721.

2. Goñi-Menoyo, José Miguel, González-Cristóbal, José Carlos, Fombella-Mourelle, Jorge: An optimised trie index for natural language processing lexicons. MIRACLE Technical Report. Universidad Politécnica de Madrid (2004).

3. Martínez, J.L., Villena-Román, J., Fombella, J., García-Serrano, A., Ruiz, A., Martínez, P., Goñi, J.M., González, J.C.: Evaluation of MIRACLE approach results for CLEF 2003. Working Notes for the CLEF 2003 Workshop, 21-22 August, Trondheim, Norway (2003).

4. Peters, C.: What happened in CLEF 2004. In: Peters, C., Clough, P., Gonzalo, J., Jones, G., Kluck M., Magnini, B. (eds.): Fifth Workshop of the Cross-Language Evaluation Forum (CLEF 2004). Lecture Notes in Computer Science (LNCS), Springer, Heidelberg, Germany (in print) (2005).

5. Peters, C., Braschler, M., Di Nunzio, G., Ferro, N.: CLEF 2004: Ad Hoc Track Overview and Results Analysis. In: Peters, C., Clough, P., Gonzalo, J., Jones, G., Kluck M., Magnini, B. (eds.): Fifth Workshop of the Cross-Language Evaluation Forum (CLEF 2004). Lecture Notes in Computer Science (LNCS), Springer, Heidelberg, Germany (in print) (2005).

6. Villena-Román, J., Martínez, J.L., Fombella, J., García-Serrano, A., Ruiz, A., Martínez, P., Goñi, J.M., González, J.C.: MIRACLE results for ImageCLEF 2003. Working Notes for the CLEF 2003 Workshop, 21-22 August, Trondheim, Norway (2003).

7. Snowball stemmers and resources. On line http://www.snowball.tartarus.org. [Visited 27/11/2004] 
8. SYSTRAN Language Translation Technlogies. On line http://www.systran.org/. [Visited 27/10/2004]

9. University of Neuchatel page of resources for CLEF (Stopwords, transliteration, stemmers ...). On line http://www.unine.ch/info/clef/. [Visited 27/10/2004]

10. Xapian: an Open Source Probabilistic Information Retrieval library. On line http://www.xapian.org. [Visited 27/10/2004]

\section{Appendix}

The appendix includes all the data that show the results obtained in the experiments. Tables 1, 3, 5, and 7 show the precisions at recall points 0 and 1 , the average precision, the percentage of the latter with respect to the best average precision experiment (the first one in each table) for each experiment. The best value is marked with the symbol "*". The comb column in each table indicates whether the experiment is a combined experiment (cf. section 4), and the sel column shows which experiments were selected for CLEF 2004, usually the ones with a better result in precision (regarding CLEF 2003 experiments).

The results for the same experiments for the CLEF 2004 campaign are also included in Tables 2, 4, 6, and 8 (the experiments submitted are indicated by the cross in the $s u b$ column), once the qrels for this campaign were made available.

Finally, Tables 9-11 show what the combined experiments consist of, as well as the type of combination used. Note that Tables 1-8 mark these with the comb column.

Table 1. CLEF 2004 results for Monolingual Russian

\begin{tabular}{|c|c|c|c|c|c|c|}
\hline at0 & at1 & avgp & $\%$ & run id & comb & sub \\
\hline 0.5707 & $0.2184^{*}$ & $0.3754^{*}$ & $0.00 \%$ & ruav5 & $\mathrm{X}$ & \\
\hline $0.5742 *$ & 0.2143 & 0.3697 & $-1.52 \%$ & runormaltit3 & & \\
\hline 0.5638 & 0.2100 & 0.3695 & $-1.57 \%$ & ruav7 & $\mathrm{X}$ & $\mathrm{X}$ \\
\hline 0.5706 & 0.2108 & 0.3685 & $-1.84 \%$ & runormaltit2 & & \\
\hline 0.5717 & 0.2080 & 0.3676 & $-2.08 \%$ & runormaltit 1 & & \\
\hline 0.5553 & 0.2092 & 0.3672 & $-2.18 \%$ & ruR1FWnormal & & $\mathrm{X}$ \\
\hline 0.5683 & 0.2014 & 0.3660 & $-2.50 \%$ & runormal & & \\
\hline 0.5693 & 0.2094 & 0.3648 & $-2.82 \%$ & rucomb1s101 & $\mathrm{X}$ & $\mathrm{X}$ \\
\hline 0.5574 & 0.2050 & 0.3641 & $-3.01 \%$ & ruav8 & $\mathrm{X}$ & $\mathrm{X}$ \\
\hline 0.5597 & 0.2094 & 0.3608 & $-3.89 \%$ & rucomb1s201 & $\mathrm{X}$ & \\
\hline 0.5558 & 0.1940 & 0.3584 & $-4.53 \%$ & ruFWnormal & & \\
\hline 0.5225 & 0.1762 & 0.3309 & $-11.85 \%$ & ruR2FWnormal & & \\
\hline 0.5102 & 0.1883 & 0.3195 & $-14.89 \%$ & rungrams54 & & \\
\hline 0.4906 & 0.1790 & 0.3125 & $-16.76 \%$ & rungrams 43 & & \\
\hline 0.4885 & 0.1771 & 0.3012 & $-19.77 \%$ & ruFWnostem & & \\
\hline 0.4731 & 0.1827 & 0.2907 & $-22.56 \%$ & rungrams76 & & \\
\hline 0.4757 & 0.1642 & 0.2884 & $-23.18 \%$ & runostem & & \\
\hline 0.1715 & 0.0128 & 0.0764 & $-79.65 \%$ & rutitngrams 43 & & \\
\hline 0.1538 & 0.0109 & 0.0723 & $-80.74 \%$ & rutitngrams54 & & \\
\hline 0.1166 & 0.0049 & 0.0433 & $-88.47 \%$ & rutitFWnormal & & \\
\hline 0.1119 & 0.0003 & 0.0383 & $-89.80 \%$ & rutitnormal & & \\
\hline
\end{tabular}


Table 2. CLEF 2003 results for Monolingual Russian

\begin{tabular}{|c|c|c|c|c|c|c|}
\hline at0 & at1 & avgp & $\%$ & run id & comb & sel \\
\hline $0.6384 *$ & 0.1459 & $0.3799^{*}$ & $-0.00 \%$ & ruav8 & $\mathrm{X}$ & $\mathrm{X}$ \\
\hline 0.6379 & 0.1465 & 0.3750 & $-1.29 \%$ & ruR1FWnormal & & $\mathrm{X}$ \\
\hline 0.6323 & 0.1471 & 0.3706 & $-2.45 \%$ & ruav7 & $\mathrm{X}$ & $\mathrm{X}$ \\
\hline 0.6344 & 0.1463 & 0.3697 & $-2.68 \%$ & ruav5 & $\mathrm{X}$ & \\
\hline 0.6234 & $0.1593^{*}$ & 0.3695 & $-2.74 \%$ & rucomb1s101 & $\mathrm{X}$ & $\mathrm{X}$ \\
\hline 0.6276 & 0.1575 & 0.3695 & $-2.74 \%$ & rucomb1s201 & $\mathrm{X}$ & \\
\hline 0.6230 & 0.1563 & 0.3695 & $-2.74 \%$ & runormaltit1 & & \\
\hline 0.6234 & $0.1593^{*}$ & 0.3695 & $-2.74 \%$ & runormaltit3 & & \\
\hline 0.6228 & 0.1585 & 0.3694 & $-2.76 \%$ & runormaltit2 & & \\
\hline 0.6254 & 0.1430 & 0.3653 & $-3.84 \%$ & ruFWnormal & & \\
\hline 0.6194 & 0.1423 & 0.3645 & $-4.05 \%$ & runormal & & \\
\hline 0.6044 & 0.1482 & 0.3605 & $-5.11 \%$ & ruR2FWnormal & & \\
\hline 0.5789 & 0.1318 & 0.3418 & $-10.03 \%$ & rungrams54 & & \\
\hline 0.5579 & 0.1438 & 0.3323 & $-12.53 \%$ & rungrams 43 & & \\
\hline 0.5609 & 0.1052 & 0.3046 & $-19.82 \%$ & ruFWnostem & & \\
\hline 0.5609 & 0.1052 & 0.3046 & $-19.82 \%$ & runostem & & \\
\hline 0.5172 & 0.1058 & 0.2753 & $-27.53 \%$ & rungrams76 & & \\
\hline 0.2922 & 0.0584 & 0.1382 & $-63.62 \%$ & ruFWtitnormal & & \\
\hline 0.2910 & 0.0584 & 0.1381 & $-63.65 \%$ & rutitnormal & & \\
\hline 0.2716 & 0.0661 & 0.1377 & $-63.75 \%$ & rutitngrams 43 & & \\
\hline 0.2378 & 0.0462 & 0.1125 & $-70.39 \%$ & rutitngrams54 & & \\
\hline
\end{tabular}

Table 3. CLEF 2004 results for Monolingual French

\begin{tabular}{|c|c|c|c|c|c|c|}
\hline at0 & at1 & avgp & $\%$ & run id & comb & sub \\
\hline 0.7070 & 0.2444 & $0.4677^{*}$ & $0.00 \%$ & frav5 & $\mathrm{X}$ & \\
\hline 0.7111 & 0.2459 & 0.4673 & $-0.09 \%$ & frcomb1s201 & $\mathrm{X}$ & $\mathrm{X}$ \\
\hline 0.7107 & 0.2438 & 0.4670 & $-0.15 \%$ & frav3 & $\mathrm{X}$ & \\
\hline 0.7100 & $0.2477^{*}$ & 0.4670 & $-0.15 \%$ & frcomb2s201 & $\mathrm{X}$ & $\mathrm{X}$ \\
\hline 0.7032 & $0.2477^{*}$ & 0.4654 & $-0.49 \%$ & frR2FWnormal & & \\
\hline $0.7242 *$ & 0.2349 & 0.4654 & $-0.49 \%$ & frFWnormal & & \\
\hline 0.6986 & 0.2459 & 0.4653 & $-0.51 \%$ & frR1FWnormal & & \\
\hline 0.6986 & 0.2459 & 0.4653 & $-0.51 \%$ & frcomb1s101 & $\mathrm{X}$ & \\
\hline 0.6998 & $0.2477^{*}$ & 0.4639 & $-0.81 \%$ & frcomb2s101 & $\mathrm{X}$ & \\
\hline 0.7170 & 0.2425 & 0.4635 & $-0.90 \%$ & frav9 & X & \\
\hline 0.7186 & 0.2338 & 0.4628 & $-1.05 \%$ & frnormalinv & & \\
\hline 0.7169 & 0.2378 & 0.4624 & $-1.13 \%$ & frav7 & $\mathrm{X}$ & $\mathrm{X}$ \\
\hline 0.7172 & 0.2352 & 0.4596 & $-1.73 \%$ & frnormal & & \\
\hline 0.7113 & 0.2371 & 0.4589 & $-1.88 \%$ & frav8 & $\mathrm{X}$ & $\mathrm{X}$ \\
\hline 0.6634 & 0.2060 & 0.4206 & $-10.07 \%$ & frngrams54 & & \\
\hline 0.6797 & 0.2036 & 0.4187 & $-10.48 \%$ & frnostem & & \\
\hline 0.6685 & 0.2014 & 0.4177 & $-10.69 \%$ & frFWnostem & & \\
\hline 0.6393 & 0.0719 & 0.3263 & $-30.23 \%$ & frtitnormalinv & & \\
\hline 0.6278 & 0.0719 & 0.3254 & $-30.43 \%$ & frtitnormal & & \\
\hline 0.6066 & 0.0619 & 0.2999 & $-35.88 \%$ & frtitngrams54 & & \\
\hline 0.5932 & 0.0650 & 0.2985 & $-36.18 \%$ & frtitnostem & & \\
\hline
\end{tabular}


Table 4. CLEF 2003 results for Monolingual French

\begin{tabular}{|c|c|c|c|c|c|c|}
\hline at0 & at1 & avgp & $\%$ & run id & comb & sel \\
\hline 0.8053 & $0.3271^{*}$ & $0.5312^{*}$ & $0.00 \%$ & frav7 & $\mathrm{X}$ & $\mathrm{X}$ \\
\hline 0.7993 & 0.2987 & 0.5288 & $-0.45 \%$ & frcomb2s201 & $\mathrm{X}$ & $\mathrm{X}$ \\
\hline 0.8091* & 0.3202 & 0.5287 & $-0.47 \%$ & frav8 & $\mathrm{X}$ & $\mathrm{X}$ \\
\hline 0.7902 & 0.3049 & 0.5220 & $-1.73 \%$ & frcomb1s201 & $\mathrm{X}$ & $\mathrm{X}$ \\
\hline 0.7707 & 0.2987 & 0.5207 & $-1.98 \%$ & frcomb2s101 & $\mathrm{X}$ & \\
\hline 0.7707 & 0.2987 & 0.5207 & $-1.98 \%$ & frR2FWnormal & & \\
\hline 0.7951 & 0.3029 & 0.5200 & $-2.11 \%$ & frav5 & $\mathrm{X}$ & \\
\hline 0.7927 & 0.3017 & 0.5191 & $-2.28 \%$ & frav3 & $\mathrm{X}$ & \\
\hline 0.7731 & 0.3049 & 0.5162 & $-2.82 \%$ & frcomb1s101 & $\mathrm{X}$ & \\
\hline 0.7731 & 0.3049 & 0.5162 & $-2.82 \%$ & frR1FWnormal & & \\
\hline 0.7954 & 0.2980 & 0.5124 & $-3.54 \%$ & frFWnormal & & \\
\hline 0.7855 & 0.2987 & 0.5083 & $-4.31 \%$ & frnormal & & \\
\hline 0.7717 & 0.2749 & 0.4913 & $-7.51 \%$ & frav9 & $\mathrm{X}$ & \\
\hline 0.7281 & 0.2958 & 0.4875 & $-8.23 \%$ & frnormalinv & & \\
\hline 0.7313 & 0.2778 & 0.4722 & $-11.11 \%$ & frngrams54 & & \\
\hline 0.6896 & 0.2753 & 0.4579 & $-13.80 \%$ & frFWnostem & & \\
\hline 0.6806 & 0.2618 & 0.4452 & $-16.19 \%$ & frnostem & & \\
\hline 0.6241 & 0.1725 & 0.3315 & $-37.59 \%$ & frtitnormal & & \\
\hline 0.5850 & 0.1516 & 0.3117 & $-41.32 \%$ & frtitngrams 54 & & \\
\hline 0.4939 & 0.1213 & 0.2288 & $-56.93 \%$ & frtitnostem & & \\
\hline
\end{tabular}

Table 5. CLEF 2004 results for Bilingual Dutch to French

\begin{tabular}{|c|c|c|c|c|c|c|}
\hline at0 & at1 & avgp & $\%$ & run id & comb & sub \\
\hline 0.5591 & 0.1716 & $0.3519 *$ & $0.00 \%$ & nl2frR2FWnormal & & \\
\hline 0.5628 & 0.1668 & 0.3505 & $-0.40 \%$ & nl2frav & $\mathrm{X}$ & $\mathrm{X}$ \\
\hline 0.5558 & 0.1637 & 0.3486 & $-0.94 \%$ & nl2frR3FWnormal & & \\
\hline 0.5458 & 0.1739 & 0.3483 & $-1.02 \%$ & nl2frR1FWnormal & & \\
\hline 0.5598 & 0.1593 & 0.3483 & $-1.02 \%$ & nl2frR5FWnormal & & $\mathrm{X}$ \\
\hline 0.5583 & 0.1595 & 0.3472 & $-1.34 \%$ & nl2frR4FWnormal & & $\mathrm{X}$ \\
\hline $0.5653^{*}$ & 0.1717 & 0.3469 & $-1.42 \%$ & nl2frnormal & & \\
\hline 0.5430 & $0.1750^{*}$ & 0.3451 & $-1.93 \%$ & nl2frFWnormal & & \\
\hline 0.5515 & 0.1595 & 0.3449 & $-1.99 \%$ & nl2frcomb1s101 & $\mathrm{X}$ & $\mathrm{X}$ \\
\hline
\end{tabular}

Table 6. CLEF 2003 results for Bilingual Dutch to French

\begin{tabular}{|c|c|c|c|c|c|c|}
\hline at0 & at1 & avgp & $\%$ & run id & comb & sel \\
\hline $0.6766^{*}$ & $0.2323 *$ & $0.4159 *$ & $-0.00 \%$ & nl2frR4FWnormal & & $\mathrm{X}$ \\
\hline 0.6564 & 0.2296 & 0.4112 & $-1.13 \%$ & nl2frR5FWnormal & & $\mathrm{X}$ \\
\hline 0.6528 & $0.2323 *$ & 0.4087 & $-1.73 \%$ & nl2frcomb1s101 & $\mathrm{X}$ & $\mathrm{X}$ \\
\hline 0.6583 & 0.2285 & 0.4069 & $-2.16 \%$ & nl2frav & $\mathrm{X}$ & $\mathrm{X}$ \\
\hline 0.6518 & 0.2286 & 0.4043 & $-2.79 \%$ & nl2frR3FWnormal & & \\
\hline 0.6684 & 0.2230 & 0.4016 & $-3.44 \%$ & $\mathrm{nl} 2 \mathrm{frFWnormal}$ & & \\
\hline 0.6423 & 0.2321 & 0.3997 & $-3.90 \%$ & nl2frR2FWnormal & & \\
\hline 0.6533 & 0.2225 & 0.3986 & $-4.16 \%$ & nl2frR1FWnormal & & \\
\hline 0.6478 & 0.2159 & 0.3862 & $-7.14 \%$ & nl2frnormal & & \\
\hline
\end{tabular}


Table 7. CLEF 2004 results for Bilingual German to French

\begin{tabular}{|c|c|c|c|c|c|c|}
\hline at0 & at1 & avgp & $\%$ & run id & comb & sub \\
\hline 0.5419 & 0.1195 & $0.3217 *$ & $0.00 \%$ & de2frR5FWnormal & & \\
\hline 0.5289 & 0.1209 & 0.3208 & $-0.28 \%$ & de2frR4FWnormal & & \\
\hline 0.5485 & 0.1263 & 0.3201 & $-0.50 \%$ & de2frR2FWnormal & & $\mathrm{X}$ \\
\hline 0.5340 & 0.1241 & 0.3199 & $-0.56 \%$ & de2frR3FWnormal & & $\mathrm{X}$ \\
\hline 0.5349 & 0.1244 & 0.3178 & $-1.21 \%$ & de 2 frav & $\mathrm{X}$ & \\
\hline 0.5439 & 0.1250 & 0.3174 & $-1.34 \%$ & de2frR1FWnormal & & \\
\hline 0.5381 & 0.1241 & 0.3166 & $-1.59 \%$ & de2frcomb1s 101 & $\mathrm{X}$ & $\mathrm{X}$ \\
\hline 0.5447 & $0.1265^{*}$ & 0.3134 & $-2.58 \%$ & de2frFWnormal & & \\
\hline 0.5265 & $0.1265^{*}$ & 0.3116 & $-3.14 \%$ & de2frcomb2s201 & $\mathrm{X}$ & $\mathrm{X}$ \\
\hline $0.5505^{*}$ & 0.1221 & 0.3100 & $-3.64 \%$ & de2frnormal & & \\
\hline
\end{tabular}

Table 8. CLEF 2003 results for Bilingual German to French

\begin{tabular}{|c|c|c|c|c|c|c|}
\hline at0 & at1 & avgp & $\%$ & run id & comb & sel \\
\hline 0.6064 & 0.2255 & $0.3999 *$ & $-0.00 \%$ & de2frR3FWnormal & & $\mathrm{X}$ \\
\hline 0.6007 & 0.2255 & 0.3975 & $-0.60 \%$ & de2frcomb1s101 & $\mathrm{X}$ & $\mathrm{X}$ \\
\hline 0.6017 & 0.2246 & 0.3942 & $-1.43 \%$ & de2frR2FWnormal & & $\mathrm{X}$ \\
\hline 0.5931 & 0.2244 & 0.3938 & $-1.53 \%$ & de2frav & $\mathrm{X}$ & \\
\hline 0.5912 & 0.2273 & 0.3931 & $-1.70 \%$ & de2frR5FWnormal & & \\
\hline 0.5867 & 0.2178 & 0.3899 & $-2.50 \%$ & de2frR1FWnormal & & \\
\hline 0.5795 & $0.2288^{*}$ & 0.3890 & $-2.73 \%$ & de2frR4FWnormal & & \\
\hline $0.6082^{*}$ & 0.2093 & 0.3837 & $-4.05 \%$ & de2frcomb2s201 & $\mathrm{X}$ & $\mathrm{X}$ \\
\hline 0.5962 & 0.2093 & 0.3816 & $-4.58 \%$ & de2frFWnormal & & \\
\hline 0.5857 & 0.2030 & 0.3770 & $-5.73 \%$ & de2frnormal & & \\
\hline
\end{tabular}

Table 9. Combined experiments for Monolingual French

\begin{tabular}{|c|c|c|c|c|c|c|}
\hline Experiment & Combination & \multicolumn{5}{|c|}{ Basic experiments } \\
\hline fr1s 101 & asym101 & frFWnormal, & frR1FWnormal & & & \\
\hline fr1s201 & asym201 & frFWnormal, & frR1FWnormal & & & \\
\hline fr2s 101 & asym 101 & frFWnormal, & frR2FWnormal & & & \\
\hline fr2s 201 & asym201 & frFWnormal, & frR2FWnormal & & & \\
\hline frav3 & average & frR2FWnormal & frFWnormal & frnormal & & \\
\hline frav5 & average & frR2FWnormal & frFWnormal & frnormal & frR1FWnormal & frnormalinv \\
\hline \multirow{2}{*}{ frav7 } & \multirow{2}{*}{ average } & frR2FWnormal & frFWnormal & frnormal & frR1FWnormal & frnormalinv \\
\hline & & frFWnostem & frngrams54 & & & \\
\hline \multirow{2}{*}{ frav8 } & \multirow{2}{*}{ average } & frR2FWnormal & frFWnormal & frnormal & frR1FWnormal & frnormalinv \\
\hline & & frFWnostem & frngrams54 & frnostem & & \\
\hline \multirow{2}{*}{ frav9 } & \multirow{2}{*}{ average } & frR2FWnormal & frFWnormal & frnormal & frR1FWnormal & frnormalinv \\
\hline & & frFWnostem & frngrams54 & frnostem & frtitnormal & \\
\hline
\end{tabular}


Table 10. Combined experiments for Monolingual Russian

\begin{tabular}{|c|c|c|c|c|c|c|}
\hline Experiment & Combination & \multicolumn{5}{|c|}{ Basic experiments } \\
\hline rucomb1s101 & asym101 & runormaltit3 & ruR1FWnormal & & & \\
\hline rucomb1s201 & asym201 & runormaltit3 & ruR1FWnormal & & & \\
\hline ruav5 & average & runormaltit3 & ruR1FWnormal & ruFWnormal & runormal & rungrams 54 \\
\hline \multirow{2}{*}{ ruav7 } & \multirow{2}{*}{ average } & runormaltit3 & ruR1FWnormal & ruFWnormal & runormal & rungrams 54 \\
\hline & & runormaltit1 & ruR2FWnormal & & & \\
\hline \multirow{2}{*}{ ruav8 } & \multirow{2}{*}{ average } & runormaltit3 & ruR1FWnormal & ruFWnormal & runormal & rungrams54 \\
\hline & & runormaltit 1 & ruR2FWnormal & runostem & & \\
\hline
\end{tabular}

Table 11. Combined experiments for Bilingual Dutch to French and German to French

\begin{tabular}{|c|c|c|c|c|c|}
\hline \multicolumn{1}{|c|}{ Experiment } & Combination & \multicolumn{3}{c|}{ Basic experiments } \\
\hline nl2frcomb1s101 & asym101 & nl2frFWnormal & nl2frR4FWnormal & \\
\hline nl2frav & average & nl2frR1FWnormal & nl2frR2FWnormal & nl2frR3FWnormal \\
\hline de2frcomb1s101 & asym101 & nl2frR4FWnormal & nl2frR5FWnormal & nl2frFWnormal \\
\hline de2frcomb2s201 & asym201 & de2frR3FWnormal & de2frR3FWnormal & \\
\hline de2frav & average & de2frR1FWnormal & de2frR2FWnormal & de2frR3FWnormal \\
\hline & & de2frR4FWnormal & de2frR5FWnormal & de2frFWnormal \\
\hline
\end{tabular}

